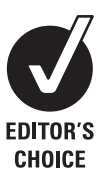

Centre for Sports and Exercise
Medicine, Barts and the London School of Medicine and Dentistry, Mile End Hospital, London, UK

Correspondence to: Dr V Tzortziou Brown, Centre for Sports and Exercise Medicine, Barts and the London School of Medicine and Dentistry, Mile End Hospital, London E1 4DG, UK; victoriagiorgio@yahoo.co.uk

Accepted 3 October 2009 Published Online First 21 October 2009

\title{
The role of the sports and exercise medicine physician in the National Health Service: a questionnaire-based survey
}

\author{
P O'Halloran, V Tzortziou Brown, K Morgan, N Maffulli, M Perry, D Morrissey
}

\section{ABSTRACT}

Objectives: To investigate the opinions of general practitioners, orthopaedic surgeons, rheumatologists, sport and exercise medicine (SEM) registrars and public health consultants on training, caseload, the most appropriate setting and the position of SEM within the National Health Service (NHS) in the UK.

Method: A cross-sectional questionnaire-based survey investigated the opinions of the above professionals from three primary care trusts and associated hospitals in London and Birmingham.

Results: With a $50 \%$ response rate $(n=226), 93 \%(208)$ 224) of participants felt that there was a role for SEM in the NHS. 56\% (124/223) agreed that this role should be in both primary and secondary care. 64\% (136/212) of participants felt that their practice would benefit from the input of a SEM physician and 95\% (191/202) would consider referring patients to SEM services. 74\% (165/ 222) agreed that SEM should have a public health role, and $63 \%$ (140/222) believed that these responsibilities should be evenly balanced with the treatment of sports injuries. Despite the emphasis on public health work from SEM policy makers, none of the SEM registrars selected public health as an important training area. 31\% (44/140) of participants felt that a lack of education in the medical profession regarding SEM represented the greatest hindrance to its development in the NHS.

Conclusion: Several areas of agreement were demonstrated across the specialties, many of which matched the views of policy makers. This study involved participants from a range of cognate disciplines and was the first to investigate this issue since SEM was recognised as a specialty in 2005 .

Sport and Exercise Medicine (SEM) was granted specialty status in the UK in 2005. ${ }^{1}$ Public health issues, such as rising obesity, and the need for specialist treatment of soft-tissue injuries have increased the necessity for these services. ${ }^{23}$ In addition, a rapidly expanding research base has enhanced the perceived credibility of the specialty, increasing the acceptance of SEM as a mainstream pursuit. ${ }^{4-6}$ The 2012 Olympics represent an unprecedented opportunity for SEM to develop in the UK. ${ }^{7-9}$ However, there is an apparent lack of a consensus on the role of SEM physicians in the National Health Service (NHS) or how these services should be delivered.

The NHS is the free-at-the-point-of-delivery system of universal health provision in the UK. Primary Care Trusts (PCTs) are local NHS organisations which provide or commission primary and community services, and are involved in commissioning secondary care. General practitioners (GPs) are primary care providers: they are usually the first point of contact for a patient. Secondary care is mainly acute (elective or emergency) care provided by medical specialists in a hospital setting. Patients are usually referred to secondary care from a primary care professional such as a GP.

The role of SEM in the NHS is a subject of much debate. $^{1011}$ Some authors have emphasised the public health responsibilities of the specialty, ${ }^{14612-15}$ and others have argued that SEM should not be seen as a quick fix for broader social problems. ${ }^{10} 1617$ Most have taken a more balanced view, ${ }^{5-9}$ 18-21 $^{2}$ and many agree that SEM faces a challenge to strike a balance between a role in treating sports injuries and its part in treating illness with exercise. ${ }^{18-10} 16-18$ This debate could affect the specialty in several ways. For example, the lack of unified objectives could limit SEM career and training pathways. Political and financial support may be allocated to cognate disciplines with more clearly defined goals, and the public perception of the function of the specialty may be confused. Cumulatively, these factors could impair the development of the specialty.

There has been little previous research on this subject, and no investigations on this particular aspect of perception and development of SEM have been conducted since specialty recognition. Thompson et $a l^{11}$ investigated the views of members of the British Association of Sports and Exercise Medicine on the desirable competencies of SEM practitioners. They reported a consensus on several attributes and on the view that SEM should have a role in the NHS, though this finding may have been influenced by participants' interest in SEM. However, the study did not expand on what this role should be.

Abernathy et a ${ }^{22}$ investigated the views of A\&E specialists regarding SEM. Knowledge of common sports injuries and the value of exercise for health were considered integral to their practice. However, there was no consensus on the most appropriate setting for SEM services. Similarly, GPs felt that the use of exercise as a health tool was vital to their practice. ${ }^{23}$ However, although participants felt undertrained to treat SEM patients, there was no agreement on whether SEM services should be integrated into the NHS.

We aimed to survey the views of healthcare professionals from several disciplines using a postal questionnaire to better explore related specialists' views on the role of SEM and therefore inform 
specialty development. ${ }^{24}$ Our primary objective was to investigate the opinions of GPs, orthopaedic surgeons, rheumatologists, SEM registrars and public health consultants on several aspects of SEM, including training, caseload, the most appropriate setting and the position of SEM within the NHS. The secondary objective was to explore whether any interdisciplinary consensus could be found regarding the most appropriate setting for SEM services in the NHS.

\section{METHOD}

\section{Questionnaire design}

A cross-sectional observational study was conducted using a self-administered postal questionnaire.

A multiple-choice questionnaire was used for data collection, and participants were given the opportunity to elaborate on their responses. The questionnaire covered several aspects of SEM to thoroughly probe participants' attitudes to the specialty and how it should integrate into the NHS.

Questions were included on participants' own practice involving respondents' attitudes to the influence of SEM on their practice, their willingness to refer patients to SEM services and potential confounders like further qualifications in SEM and years of experience. To explore how healthcare professionals would like to interact with the specialty, the participants were asked for their opinions on the most appropriate setting for SEM services and whether there was a role for SEM in the NHS. There was a question on SEM caseload to investigate views on how resources should be split between Public Health and sports injury work. Finally, questions were included regarding training to explore opinions on what competencies SEM physicians should have and whether or not experience of participating in sport was necessary.

The questionnaire was piloted on a small number of GPs from Tower Hamlets (TH) PCT, with no changes suggested. A first phase of data collection was completed between January and May 2008. Some minor modifications were made to the questionnaire before a second phase of data collection was carried out and completed in May 2009. These modifications included the addition of a question concerning the most appropriate primary care setting for SEM and the alteration of a question regarding SEM training to include the areas covered in the higher specialist training (HST) curriculum. ${ }^{25}$

\section{Questionnaire administration and data analysis}

The questionnaire was sent by post with a covering letter and a prepaid self-addressed envelope. It was explained that consent was implied in returning the questionnaire. In addition, participants were informed that their answers would be treated anonymously. ${ }^{26}$ Participants were offered a summary of the results, and non-responders were contacted again after 4 and 8 weeks. The national research ethics service was contacted and confirmed that ethical approval was not required.

Participants were drawn from South Birmingham (SB), Richmond and Twickenham (RT) and TH PCTs, and associated secondary care trusts. These three geographically diverse areas were studied to try to enhance the generalisability of the results. The participants were GPs, and orthopaedic and rheumatology consultants. These groups were selected, as they are likely to have the most interaction with SEM services. SEM registrars were also included. In addition, public health consultants from each PCT were included. Participants were identified by contacting the PCT or secondary care trust and checked against details provided on the NHS Choices website. ${ }^{27}$ The questionnaires sent to these groups were similar with slight variations in the wording to reflect specialty specifics. For example, questions regarding the referral of patients to SEM services were excluded from the public health version. In order to maximise participation, no exclusion criteria were applied.

The formulae proposed by Dilman ${ }^{28}$ and Cochran ${ }^{29}$ were used to perform sample size calculations. The calculations assumed a $55 \%$ response rate, in accordance with the response rates found in previous studies. ${ }^{1122}$ Thus, the total sample for the study included 330 GPs, 108 consultants and 15 registrars. The GPs were selected by random-number allocation. Given the small population, all of the consultants and registrars were included.

The data were analysed using SPSS 17.0 (SPSS, Chicago). Initially, proportions of responses for each question were calculated with their CIs.

The second stage involved comparing responses between specific groups within each of the following categories: specialty, PCT, additional qualifications in SEM and years of experience. PCTs were split into London and Birmingham groups, years of experience into $<5$ and $\geqslant 5$ years groups and specialties were compared with GPs as a control. For each category, $\chi^{2}$ tests were used to assess the relationship between the groups and responses $(\alpha=0.05)$. In the case of specialty, where there were more than two groups, further posthoc $\chi^{2}$ analyses were performed between the three possible paired group comparisons. In this case, a Bonferroni correction was performed to account for repeated testing $(\alpha=0.017)$. Where the frequency of responses was insufficient, the Fisher exact test was used. ${ }^{30-32}$ To enable an effective analysis, the response categories were collapsed into manageable numbers of responses. Similarly, regarding the most appropriate secondary care setting for SEM, answers were combined to represent traditional settings versus independent SEM departments.

\section{RESULTS}

\section{Overview}

A total of 226 responses (50\%) were received (table 1). Particularly high return rates were seen from public health consultants and SEM registrars. Response rates were fairly consistent between PCTs, with the exception of orthopaedic and rheumatology consultants. In this group, the response rate from $\mathrm{TH}$ was nearly double that of RT and SB, possibly reflecting differences in SEM provision.

\section{Participants' practice}

Fifty-eight per cent (130/223) of respondents had over 10 years of experience in their specialty, and 11\% (24/223) had less than 2 years. SEM registrars $\left(\chi^{2}=34.78, p<0.01\right)$ and public health consultants $\left(\chi^{2}=21.22, p<0.01\right)$ had significantly fewer years compared with GPs. In addition, London-based respondents had significantly fewer years of experience than Birmingham participants $\left(\chi^{2}=7.04, \mathrm{p}<0.01\right)$. Only $17 \%(36 / 212)$ of participants had undertaken further training in SEM.

Sixty-four per cent $(136 / 212)$ of participants felt that their practice would benefit from the input of a SEM physician. There was an agreement between the disciplines on this issue. Fourteen per cent (29/212) already had this input, though interestingly this figure was higher in consultants compared with GPs.

Ninety-five per cent (191/202) of respondents would consider referring patients to SEM services. Fifty per cent (110/222) believed that patients should be able to self-refer to SEM 
Table 1 Response rates by specialty and primary care trust

\begin{tabular}{lccc}
\hline & Total no in sample & Total no of responses & Response rate (\%) \\
\hline GPs (total) & 330 & 154 & 47 \\
TH & 115 & 54 & 47 \\
RT & 93 & 42 & 45 \\
SB & 58 & 48 \\
Orthopaedic and rheumatology & 97 & 53 & 55 \\
consultants (total) & & & \\
TH & 23 & 20 & 87 \\
RT & 9 & 45 \\
SB & 20 & 24 & 44 \\
Sport and exercise medicine registrars & 54 & 10 & 67 \\
Public health consultants (total) & 15 & 9 & 82 \\
TH & 11 & 3 & 75 \\
RT & 4 & 3 & 100 \\
SB & 3 & 3 & 75 \\
Total & 4 & 226 & 50 \\
\hline
\end{tabular}

services, while 41\% (90/222) disagreed. Again, in both these questions, the results were similar across the specialties.

\section{Caseload}

Seventy-four per cent (165/222) of respondents felt that SEM should have a public health role. Sixty-three per cent (140/222) believed that these responsibilities should be evenly balanced with the treatment of sports injuries. This view was consistent across the specialties.

\section{Training}

Fifty-five per cent (122/223) of respondents felt that it was important for SEM physicians to have experience of participating in sport, while 28\% (63/223) disagreed. This pattern was seen in each specialty except public health, where most respondents felt that it was unimportant, perhaps reflecting differences in the experience required for the public health and injury management roles of SEM. Participants were also asked to select three areas in which they felt SEM physicians should receive training (fig 1). Several further areas were suggested, including dietetics, podiatry and psychiatry. The low number of $A \& E$ and public health responses overall was noteworthy. In addition, more SEM registrars selected A\&E than in the other specialties, and no registrars selected public health.

\section{Setting}

Ninety-three per cent (208/224) of participants felt that there was a role for SEM in the NHS. Fifty-six per cent (124/223) felt that SEM had a role in both secondary and primary care. Both findings were seen consistently across the specialties.

Fourty-four per cent (95/216) of respondents felt that orthopaedics or rheumatology was the most appropriate secondary care setting for SEM, and 36\% (78/216) favoured an independent SEM department. London-based participants were more likely to favour independent SEM departments $\left(\chi^{2}=11.22, \mathrm{p}<0.01\right)$ compared with Birmingham participants, perhaps reflecting differences in the provision of services between the regions. Likewise, SEM registrars were more likely
Figure 1 Summary of the priorities for sport and exercise medicine physicians' training from all participants.

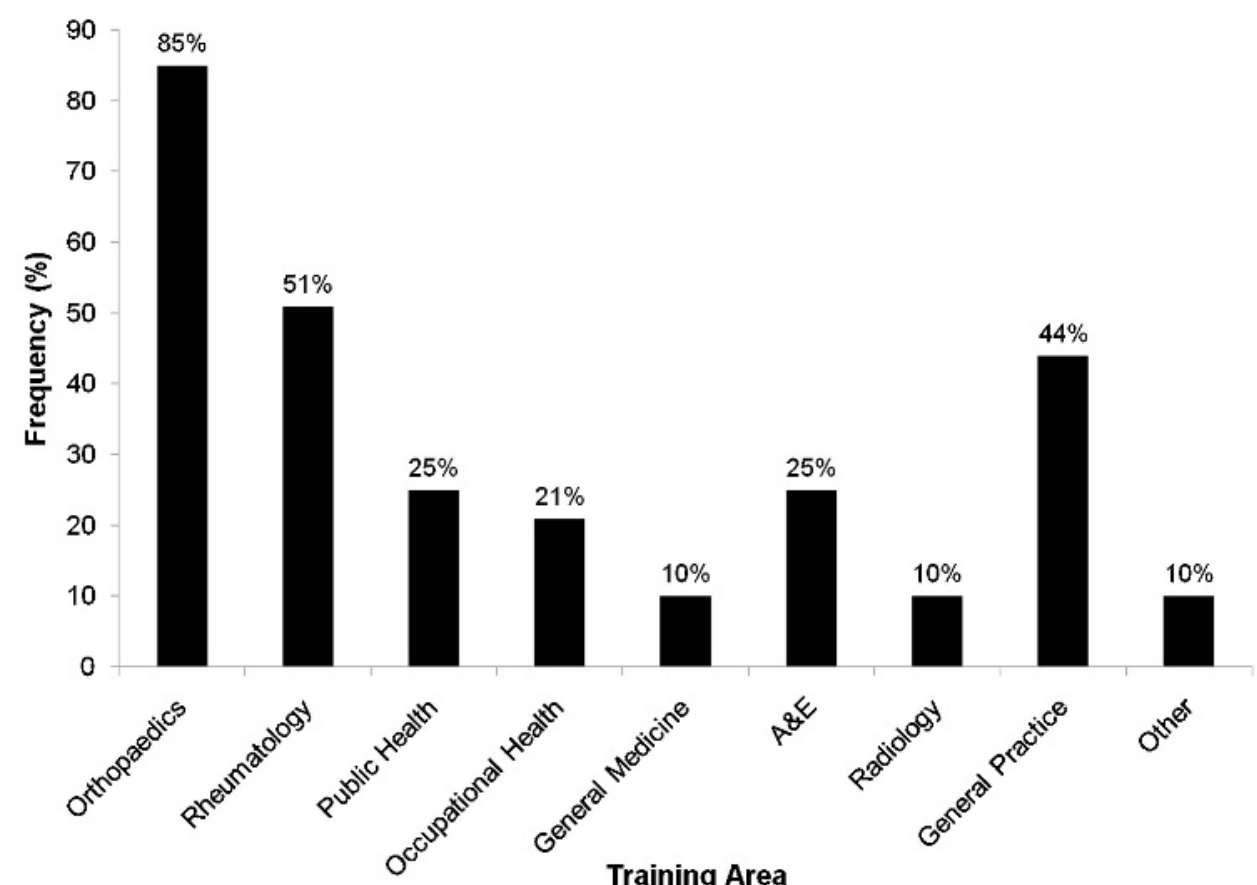


Figure 2 Participants' views regarding the primary care setting for sport and exercise medicine by specialty.

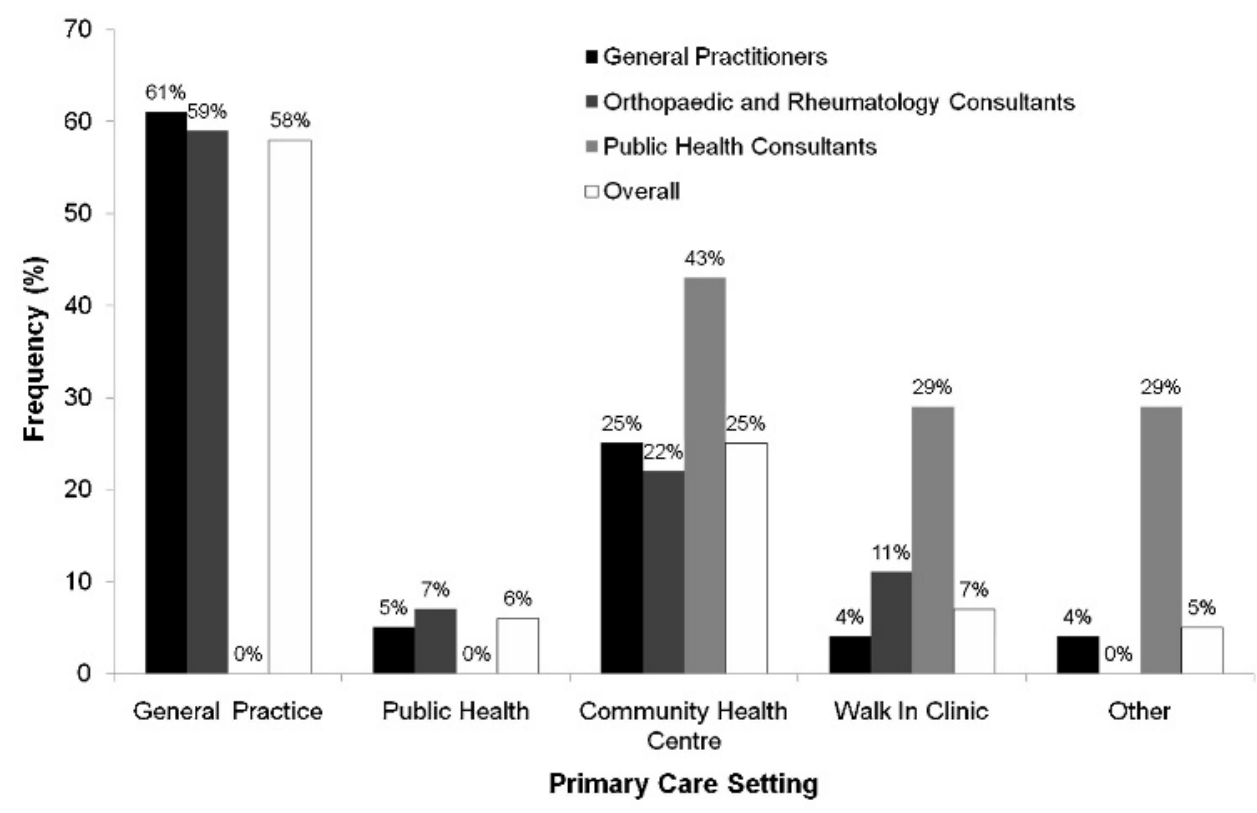

to favour independent provision compared with GPs $\left(\chi^{2}=14.21, p<0.01\right)$.

Fifty-eight per cent $(73 / 127)$ of participants felt that General Practice was the most appropriate primary care setting (fig 2), with public health consultants favouring alternatives to general practice, though this was not significant. It was also noteworthy, considering the number of respondents who felt that SEM should have a public health role, that very few considered public health an appropriate primary care setting. In addition, none of the public health consultants felt that public health would be an appropriate setting.

Participants were also asked what factors they felt hindered the development of SEM, shown in fig 3. Insufficient funding and inadequate education in the medical profession regarding the role of SEM were seen as most significant. These views were consistent between specialties. This question produced several further comments from respondents. One felt that a "lack of recognition of the ... importance of ... exercise amongst the public and politicians" was a significant barrier; another that the "exercise culture" in the UK was an issue.

\section{DISCUSSION}

We investigated the opinions of a range of healthcare professionals on several aspects of SEM. There are several areas of agreement between the specialties. Respondents agreed that SEM should have public health responsibilities and that these duties should be evenly split with sports injury work. To our knowledge, this is the first time that this issue has been studied. In addition, there was some agreement across the specialties on the most appropriate setting. In contrast with some previous work, ${ }^{23}$ respondents agreed that there was a role for SEM in the NHS. Most participants believed that this should be in both
Figure 3 Summary of perceived hindrances to the development of sport and exercise medicine (SEM) in the NHS for all participants.

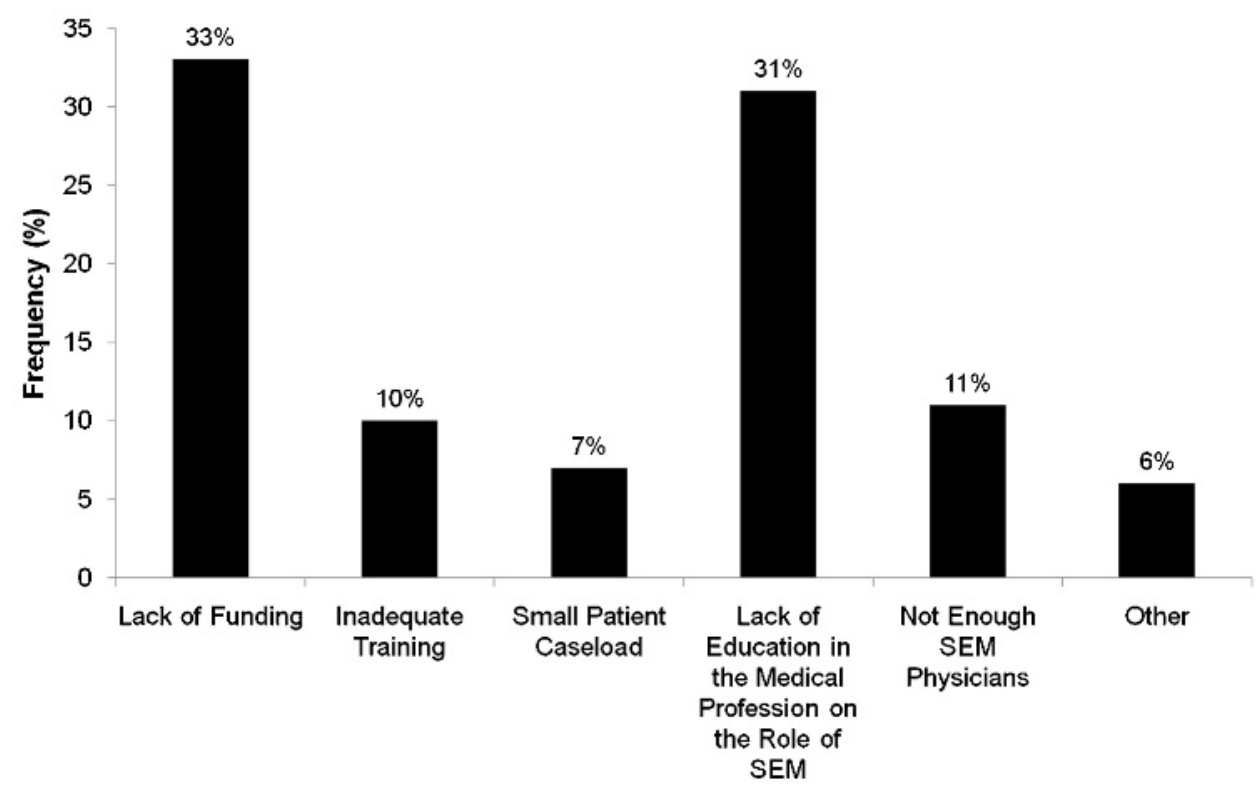

Factor 
primary and secondary care, and that in primary care SEM belonged to general practice.

Most participants felt that their practice would benefit from SEM input and would consider referring patients to SEM services. This is noteworthy, first because it indicates an increased acceptance of SEM into the mainstream but also because, as discussed in the application for specialty status, policy makers view the formation of synergistic relationships between SEM and these specialties as essential. ${ }^{25}{ }^{25}$ Several other areas of agreement also showed similarities to the literature from policy makers. Documents from the Faculty of SEM, including the HST curriculum, recognised the importance of a balance between public health and sports injury work, for example, and acknowledged the role of SEM in both primary and secondary care. ${ }^{25} 3334$ These similarities provided evidence of a pathway for the development of the specialty.

Some differences of opinion were also found. There was no agreement on the most appropriate secondary care setting for SEM. Respondents were split between traditional settings and independent SEM departments. The regional variation regarding this question may change over time as SEM provision becomes more widespread in terms of facilities and training positions. A split was also seen regarding whether patients should be able to self-refer to SEM services. Fifty per cent of participants felt that self-referral was appropriate, while $41 \%$ disagreed, including one participant who believed that sports people were "paranoid and would swamp the service."

The areas in which SEM physicians should receive training also demonstrated some interesting variation. Despite the HST curriculum including an independent set of public health competencies, ${ }^{25}$ none of the SEM registrars selected this as an important training area. Training in emergency medicine was also given little importance in contrast to previous findings. ${ }^{11}$ Sporting experience was considered important, again in contrast to previous work. ${ }^{11}$

Regarding the issue of hindrances to the development of SEM, the most commonly selected factor was inadequate funding. The second was a lack of education in the medical profession regarding the role of SEM. However, this is hardly surprising considering the indecision shown within the specialty itself. Regarding the latter, one participant commented: "There is no ... definition of what it could treat (or) the synergies with other professions." Another felt: "There is a perception this role is already covered by other disciplines."

Thus, based on these findings, SEM should be available in the NHS in both general practice and as part of hospital-based orthopaedic and rheumatology departments. SEM physicians, who should be trained in these traditional specialties, should be involved in both the treatment of sporting injuries and the use of exercise to treat illness in the clinical setting. Although this is a helpful model, it presents some practical complications. For example, the limited willingness to involve SEM in public health departments may restrict the efficacy of SEM in this area. In addition, the issue of referral is crucial to ensure that the primary and secondary care forms of the specialty are able to work synergistically.

The study presents several limitations. Participants were drawn from three PCTs, limiting the generalisability of the findings. However, this was unavoidable as a nationwide survey was beyond the scope of this study. In addition, the geographic and demographic diversity of the areas studied helps preserve the validity of the findings. The small numbers of public health consultants and SEM registrars in these areas also limited the generalisability of the findings from these populations but reflect the small numbers available. Issues such as responder bias were also likely to have affected the results. The limited qualitative data in this study is also an area for future improvement.

However, despite these limitations, this study provides a comprehensive platform for further research in several areas. The issue of self-referral and the setting for SEM services warrants further investigation to develop pathways for doctors and patients to access SEM services effectively. Similarly, the issue of hindrances to the development of the specialty requires exploration. While funding may improve in the approach to the London Olympics, the issue of education in the profession requires further attention to prevent SEM from being perceived by healthcare professionals as superfluous. The disparity between the aims of policy makers, as demonstrated in the HST curriculum, ${ }^{225}$ and those of SEM physicians also requires further examination, particularly following specialty recognition, to ensure the aims of these groups are unified. An audit of the utilisation of SEM services by both patients and the specialties surveyed here would also provide useful information to demonstrate how these opinions translate into practice.

\section{CONCLUSION}

This study investigated the opinions of a range of healthcare disciplines on several aspects of SEM and its position within the NHS and demonstrated that agreement could be found regarding several issues between these disciplines. This was one of the largest studies of its kind, both in scale and in the variety of specialists surveyed, and the first to investigate this topic since specialty recognition in the UK in 2005.

\section{Competing interests: None.}

Provenance and peer review: Not commissioned; externally peer reviewed.

\section{REFERENCES}

1. Cullen M, Batt M. Sports and exercise medicine in the United Kingdom comes of age. Br J Sports Med 2005;39:250-1

2. Faculty of Sports and Exercise Medicine. Developing specialties in medicine-the case for recognition of sport and exercise medicine as a new CCST (CCT) specialty. http://www.fsem.co.uk/DesktopModules/Documents/DocumentsView.aspx?tablD = 0yltemlD=31928\&Mld =5261\&wversion=Staging (accessed 19 April 2009)

3. Abernathy L, McNally 0, MacAuley D, et al. Sports medicine and the accident and emergency medicine specialist. Emerg Med J 2002;19:239-41.

4. Iliffe S, Masud T, Skelton D, et al. Promotion of exercise in primary care. BMJ 2008;337:a2430.

5. Lebrun C. Sports medicine: not "just for jocks" anymore. Can Fam Physician 2006:52:703-4

6. Sallis R. Exercise is medicine and physicians need to prescribe it! $B r \mathrm{~J}$ Sports Med 2009;43:3-4.

7. Batt $\mathbf{M}$, Maryon-Davis A. Sports and exercise medicine: a timely specialty development. Clin J Sports Med 2007;17:85-6.

8. Till SH, Batt M. Opening the chamber of secrets: our response to "The end of the beginning." Br J Sports Med 2006;40:566.

9. Verow P. Sports and occupational medicine: two sides of the same coin? Occup Med (Chic III) 2006;56:224-25.

10. McCrory P. What is sports and exercise medicine. Br J Sports Med 2006:40:955-7.

11. Thompson B, MacAuley D, McNally 0 , et al. Defining the sports medicine specialist in the United Kingdom: a Dephi study. Br J Sports Med 2004;38:214-17.

12. Calman K, Waring M, Warburton P. Role of sport and exercise medicine in the NHS Br J Sports Med 1999;33:374.

13. Orchard J, Brukner P. Sport and exercise medicine in Australia. Med J Aust 2005; 183:383.

14. Orchard J, Leeder S, Moorhead G, et al. Australia urgently needs a federal government body dedicated to monitoring and preventing sports injuries. Med J Aust 2007:187:505-6.

15. Orchard J. Preventing sports injuries at the national level: time for other nations to follow New Zealand's remarkable success. Br J Sports Med 2008;42:392-3.

16. McCrory P. The end of the beginning. Br J Sports Med 2006:40:1.

17. McCrory P. The reformation of sports medicine. Br J Sports Med 2007:41:281-2.

18. Batt M, Macleod D. The coming of age of sports medicine. BMJ 1997;314:621.

19. Humphries D, Milne C. Specialist status: holy grail, poisoned chalice, or flat cola? Clin J Sports Med 2008:18:109-10. 
20. Puffer J. Sports medicine: the primary care perspective. South Med J 2004;97:873-4.

21. Wilk V, Abrams D. Sports medicine meets musculoskeletal medicine. Aust Fam Physician 2007:36:385-480.

22. Abernathy L, MacAuley D, McNally 0, et al. How important is sport and exercise medicine to the accident and emergency specialist? A study in the UK and Ireland. Emerg Med J 2003;20:540-2.

23. Buckler D. General practitioners' training for, interest in, and knowledge of sports medicine training and its organisations. Br J Sports Med 1999;33:360-3.

24. Meadows K. So you want to do research? 5: questionnaire design. Br J Community Nurs 2003;8:562-70.

25. Faculty of Sports and Exercise Medicine. Specialty Training Curriculum for Sports and Exercise Medicine. http://www.fsem.co.uk/DesktopModules/Documents/ DocumentsView.aspx?tablD =0\&ltemID =31930\&Mld =5261\&wversion= Staging (accessed 19 April 2009).

26. Gillham B. The pros and cons of questionnaires. In Gillham B. Developing a questionnaire. 2nd edn. London:Continuum, 2008:1-14.

27. Department of Health. NHS Choices. http://www.nhs.uk/Pages/HomePage.aspx (accessed 19 June 2009).
28. Dilman D. Reduction of coverage and sampling errors. In: Dilman A, ed. Mail and internet surveys: the tailored deisng method. 2nd edn. New York: John Wiley, 1999:194-215.

29. Cochran W. The estimation of sample size. In: Cochran W, ed. Sampling techniques. 2nd edn. New York: John Wiley, 1963:72-8.

30. Bowers D. Testing hypothesis about the equality of population proportions. In: Bowers D, ed. Medical statistics from scratch. 1st edn. London: John Wiley, 2002:142-52.

31. Fink A. Selecting commonly used statistical methods for surveys. In: Fink A, ed. How to analyse survey data. 1st edn. USA: Sage, 1995:49-78.

32. Petrie A, Sabin C. Basic techniques for analysing data. In: Petrie A, Sabin C, eds. Medical statistics at a glance. 2nd edn. Oxford: Blackwell, 2005:46-101.

33. Faculty of Sports and Exercise Medicine. The role of the specialist doctor in sports and exercise medicine. http://www.fsem.co.uk/DesktopModules/Documents/ DocumentsView.aspx?tablD=0\&ltemID =114445\&Mld =5288\&wversion= Staging (accessed 19 April 2009).

34. Faculty of Sports and Exercise Medicine. ISEM vision statement. http://www fsem.co.uk/DesktopModules/Documents/DocumentsView.aspx?tablD=0\&ltemID = $114354 \&$ Mld $=5261$ \&wversion = Staging (accessed 19 April 2009). 